\title{
Ethnicity Variation in the Treatment of Pediatric Lupus Nephritis
}

\author{
Aanchal Shrestha ${ }^{1}$, Ruochen Che $^{1}$ and Zhang Aihua ${ }^{* 1,2}$ \\ ${ }^{1}$ Department of Nephrology, Children's Hospital of Nanjing Medical University, Nanjing 210008, China \\ ${ }^{2}$ Nanjing Key Laboratory of Pediatrics, Children's Hospital of Nanjing Medical University, Nanjing 210008, China
}

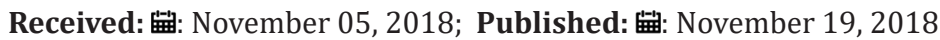

*Corresponding author: Aihua Zhang, Department of Nephrology, Children's Hospital of Nanjing Medical University, China

\begin{abstract}
Systemic lupus erythematosus (SLE) is known as a complex autoimmune disorder which is characterized by chronic systemic inflammation resulting from antibodies directed against self-antigens, immunity dysfunction and immune complex formation affecting multi-organ system. Lupus nephritis (LN) is a serious component of SLE in which $40-80 \%$ are diagnosed in childhood and exerts adverse impact on long-term renal and patient survival. African-Americans (AA), Hispanics, Asians, and non-Caucasians are high-risk populations with higher prevalence and severe disease. Despite the similarities in adults, childhood-onset LN tends to have more active onset and severe disease activity requiring timely recognition and proper treatment. Over the past 2 decades, huge progress has been made in the treatment of childhood LN. Ethnicity has a quite well-defined effect on the response to treatment for which special considerations must be taken in treatment strategy. This review will discuss the current therapies of $\mathrm{LN}$ in clinical practice and shows a comparison of treatment responses in different ethnicities.
\end{abstract}

Keywords: Lupus Nephritis; Systemic Lupus Erythematosus; Pediatric; Ethnicity; Immunosuppressive Treatment

Abbreviations: SLE: Systemic Lupus Erythematosus; LN: Lupus Nephritis; AA: African-Americans; CYC: Cyclophosphamide; NIH: National Institutes of Health; IV: Intravenous; ELNT: Euro-Lupus Nephritis Trial; MMF: Mycophenolate Mofetil; RCT: Randomized Controlled Trial; ALMS: Asperva Lupus Management Study; AZA: Azathioprine; CNI: Calcineurin Inhibitors (CNIs); RTX: Rituximab; HCQ: Hydroxychloroquine; ACR: American College of Rheumatology; KDIGO: Kidney Disease Improving Global Outcomes; EULAR: European League Against Rheumatism

\section{Introduction}

About $10 \%$ to $20 \%$ of SLE cases are diagnosed during childhood which uses 18 years as the upper cutoff age in most of the studies [1]. The kidney involvement i.e. lupus nephritis (LN) is a serious component of SLE which presents with proteinuria, microscopic hematuria and renal dysfunction thereby exerting adverse impact on long-term renal and patient survival. Up to 40 to $80 \%$ of SLE children will develop LN during their disease course. The incidence rates per 100,000 Medicaid-Enrolled children per year for LN was estimated to be higher in Asians (2.08), African-Americans (AA) (0.87) and Hispanics (0.85) as compared with Whites (0.30) [2]. In the same study, prevalence rates per 100,000 children per year are higher among Asians (11.21), AA (5.79), Hispanics (4.30) as compared with Whites (1.19). LN in children, in contrast to adultonset, have earlier and more aggressive disease activity with high prevalence and relatively poor response to treatment which leads to poor quality of life [3-5]. With the use of standardized treatment based on guidelines, response to treatment still shows variation between different ethnicities. Ethnicity has a major effect in the disease prevalence, clinical manifestations, severity, and response to treatment in SLE indicating that AA, Hispanics, Asians, and non-Caucasians are more prone to the disease severity [6-8]. This review summarizes the current treatment of $\mathrm{LN}$ in clinical practice and compares the responses in different ethnic groups.

\section{Treatment}

Historically, patients with LN were treated with low-dose steroids alone. Before the initiation of immunosuppressive regimens, a study showed that a two-year survival rate was less than $10 \%$ in patients with diffuse proliferative glomerulonephritis [9]. The standard treatment regimens for pediatric LN have been mostly derived from the studies in adults which has progressed over the few past decades with the usage of corticosteroids alone to the combination of different immunosuppressant drugs resulting improvements in overall patients and renal survival. The early treatment of acute lesions greatly contributes to delay disease 
progression and achieve remission hence improving the survival rates of patients with LN [10]. The treatment of lupus nephritis involves two phases: an induction phase, in order to reverse the immune-mediated inflammatory processes with the use of potent immunosuppressive medications. This is followed by a maintenance phase, in which a longer period of lower dose immunosuppressive medications is used in order to maintain a stable state and prevent recurrences.

\section{Cyclophosphamide (CYC)}

With the advent of CYC in the treatment of LN in children, renal survival at 5 and 10 -year was significantly better [11]. The landmark National Institutes of Health (NIH) study led to the routine use of high dose intravenous (IV) CYC plus prednisolone as induction therapy which was superior to the pulse steroids alone and it became the 'standard of care' [12]. Several studies with the use of high dose CYC in children with different ethnicities showed good outcome [13-16]. Despite the good outcome, IV CYC treatment is associated with short- and long-term side effects, such as infections, alopecia, nausea, amenorrhea, infertility and hemorrhagic cystitis. In order to reduce the CYC toxicity, low-dose intermittent IV CYC was investigated. The Euro-Lupus Nephritis Trial (ELNT) on adult population compared a high dose NIH regimen of IV CYC (six monthly pulses at a dose of $0.5-1 \mathrm{mg} / \mathrm{m} 2$ followed by two quarterly pulses) with the Euro-Lupus low dose regimen (six pulses of IV CYC every two weeks at a fixed dose of $500 \mathrm{mg}$ ). ELNT with reduced CYC dose has been shown to be as effective as the standard dose CYC regimen when used in $85 \%$ of the patient being Caucasians with less severe proliferative LN and it was associated with fewer side effects [17]. A little data has been reported on the efficacy of the Euro-Lupus regimen in Asians and non-Caucasians. So, there still needs to be demonstrated that a low-dose CYC regimen can be effectively used in patients with other ethnicities including AA, Hispanics, and Asians.

\section{Mycophenolate Mofetil (MMF)}

Based on the studies reported in adult patients, from the beginning of the 21st century the use of MMF as induction therapy and maintenance therapy is increasing in children and signifies a major progress in the immunosuppressive therapy for severe LN. The first randomized controlled trial (RCT) done in an adult Chinese population with diffuse proliferative LN comparing oral CYC (2.5 mg/kg/day) with MMF (1 gm twice daily) as induction therapy showed equivocal improvement in complete renal and relapse rates. Furthermore, patients treated with MMF had fewer adverse events including infections, alopecia, and amenorrhea as compared with CYC [18]. Likewise, several data on Asian children show no significant differences in the efficacy between MMF and CYC [19-21]. In a sub-analysis of the Asperva Lupus Management Study (ALMS), the response variation with race and ethnicity was demonstrated, in that Asians and Whites had a similar response to CYC and MMF whereas Black and Hispanics showed the better result with MMF (60.4\%) compared to CYC (38.5\%) [22]. The retrospective cohort data on children (56\% AA, 29\% Hispanics) as per treatment protocol was compared which showed the 5-year renal survival was 91\% in MMF group compared to 52\% in the CYC group $(\mathrm{P}<0.01)$ [23]. The MAINTAIN Nephritis Trial established no significant difference (in terms of renal outcomes and time to disease flare) between MMF (2 gm/day) and AZA (2 mg/kg/day) as maintenance therapy in (mostly) white Europeans [24], and the similar result in Whites was reported in the 10-year follow-up of the MAINTAIN Nephritis Trial [25]. However, the maintenance phase of the ALMS with a much greater proportion of nonCaucasians showed the superiority of MMF over azathioprine for the prevention of renal relapses [26].

\section{Azathioprine (AZA)}

AZA is another commonly used immunosuppressive regimen in a maintenance therapy of LN. A report on Chinese patients showed treatment with either MMF as both induction and maintenance phase or sequential CYC followed by AZA regimen showed comparable long-term efficacy in the renal preservation and renal outcome [18]. Similarly, in high-risk AA and Hispanics, short-term CYC induction therapy which is followed by either MMF or AZA maintenance showed equal efficacy [27]. Use of AZA in a multiethnic cohort study of children proved excellent long-term outcome which further suggested that Non-Caucasians patients may be at increased risk for renal failure when compared to Caucasians [28].

\section{Calcineurin inhibitors (CNIs)}

CNIs has a primary effect on T-cell expansion and has been used as a potent immunosuppression in the treatment of proliferative LN especially in Asians. Subsequent studies in Chinese patients showed equal efficacy between tacrolimus, IV CYC or MMF [29,30]. The data on long-term use of tacrolimus in Japanese children at a low dose of $0.03-0.075 \mathrm{mg} / \mathrm{kg} /$ day along with a tapering dose of steroid with a mean treatment duration for 42 months showed the treatment is beneficial with low cytotoxicity [31]. A small randomized study on Taiwanese children revealed equivocal improvements in renal outcomes treated with cyclosporine A and steroids when compared with oral CYC [32]. A large study in Chinese patients with class III/IV with or without concomitant class V LN under multi-target induction therapy with tacrolimus ( $4 \mathrm{mg} /$ day), MMF (1 gm/day) and steroid showed higher complete remission rate (45.9 vs. $25.6 \%$, $\mathrm{p}<0.001)$ and overall response rate ( 83.5 vs. $63.0 \%, \mathrm{p}<0.001)$ when compared with IV CYC and corticosteroids, while the incidence of adverse events did not differ between the two regimens [33]. These patients were further continued to receive maintenance therapy with either multi-target regimen (tacrolimus+ MMF+ steroid) or AZA, resulted in a low renal relapse rate and fewer adverse events in the multi-target group [34]. Data from Asians show that multi-target therapy in patients with pediatric-onset LN can be a promising therapeutic option for introducing and maintaining remission in severe proliferative LN [35,36]. Multi-target therapy in $\mathrm{AA}$ and Caucasians with tacrolimus and MMF in MMF-resistant cases showed effective outcome $[37,38]$.

\section{Rituximab (RTX)}

RTX is a specific monoclonal antibody against human B-cell CD20 receptor which has emerged as a salvage therapy mostly confined to those patients who have resistant or relapsing proliferative 
nephritis. There are numerous case series in children demonstrating the usefulness of RTX [39-41]. In a study of childhood-onset LN (mostly Africans) who were treated with RTX $375 \mathrm{mg} / \mathrm{m} 2$ once a week for two to four weeks, $93 \%$ of patients reportedly achieved improvement in disease activity. However, the safety of RTX remains unanswered [39]. In contrast, the retrospective review in children with most of them being AA and Hispanics failed to show any improvement in renal survival when treated with RTX compared to MMF [23]. Adverse events like infusion reactions, anaphylaxis, hematologic toxicity, infections, hypogammaglobulinemia, and progressive multifocal leukoencephalopathy may be seen with RTX treatment. Long-term observational data and large RCTs with the use of RTX in multiethnic groups are warranted in order to identify the response variation and verify its long-term safety profile.

\section{Adjunctive Therapy}

Antimalarial drug such as hydroxychloroquine (HCQ) as an adjunctive therapy has been recommended in the American College of Rheumatology (ACR), Kidney Disease Improving Global Outcomes (KDIGO) and European League against Rheumatism (EULAR) guidelines for long-term management of lupus nephritis in all patients, unless there is a contraindication because it reduces incidence of renal flares, renal injury, accrual or organ damage, thrombosis and increases long-term survival [42-44]. The result from LUMINA study, a multiethnic cohort of AA, Hispanics and Caucasians demonstrated the reduced occurrence of renal damage and lower disease activity with the use of HCQ [45]. Recently, a large study of SLE patients (93\% Caucasians) showed an inverse relationship between the time of exposure to antimalarial drug and risk of infection [46]. The usefulness of HCQ has been studied in lupus patients of all ethnicities [47]. Medicines to control blood pressure using angiotensin-converting enzyme inhibitors and angiotensin receptor blockers are not only for hypertension but also plays a crucial role in reducing proteinuria, use of anticoagulants and lipid-lowering drugs which are the mainstay of adjunctive therapy. In addition, calcium and vitamin D supplements are also beneficial. Infection is the commonest cause of death in childhood SLE, therefore children on immunosuppression should be closely monitored.

\section{Conclusion}

Treatment in LN with advances in immunosuppressive regimens has provided better control of disease activity, good disease response and considerable improvement in overall patient survival rates. However, there are still variations of therapy responses between different races. $\mathrm{LN}$ is less common in Caucasians SLE patients with less severity and better outcome compared to AA, Hispanics, and Asians. Thus, Caucasians might get more benefit from relatively low-dose regimens. However, largescale studies and RCTs in children comparing treatment strategies according to ethnicities are warranted in order to provide safe and adequate treatment. Therefore, the role of ethnic variation in response to treatment and eventual prognosis in children require much attention and research in the future.

\section{Acknowledgment}

This work was supported by grants from National Natural Science Foundation of China (Nos. 81830020, 81873599, 91742205, 81625004, 81670678, 81530023 and 81470928), the National Key Research and Development Program (No. 2016YFC0906103), and the Natural Science Foundation of Jiangsu Province (Nos. BK20141079, and BL2014007).

\section{References}

1. Silva CA, Avcin T, Brunner HI (2012) Taxonomy for systemic lupus erythematosus with onset before adulthood. Arthritis care \& research 64(12): 1787-1793.

2. Hiraki LT, Feldman CH, Liu J, Alarcón GS, Fischer MA, et al. (2012) Prevalence, incidence, and demographics of systemic lupus erythematosus and lupus nephritis from 2000 to 2004 among children in the US Medicaid beneficiary population. Arthritis and rheumatism 64(8): 2669-2676.

3. Carreño L, López-Longo FJ, Monteagudo I, Rodríguez Mahou M, Bascones $M$, et al. (1999) Immunological and clinical differences between juvenile and adult onset of systemic lupus erythematosus. Lupus 8(4): 287-292.

4. Ambrose N, Morgan TA, Galloway J, Ionnoau Y, Beresford MW, et al. (2016) Differences in disease phenotype and severity in SLE across age groups. Lupus 25(14): 1542-1550.

5. Brunner HI, Gladmann DD, Ibanez D, Urowitz MD, Silverman ED (2008) Difference in disease features between childhood-onset and adult-onset systemic lupus erythematosus. Arthritis Rheum 58(2): 556-562.

6. Tucker LB, Uribe AG, Fernández M, Vilá LM, McGwin G, et al. (2008) Adolescent onset of lupus results in more aggressive disease and worse outcomes: results of a nested matched case-control study within LUMINA, a multiethnic US cohort (LUMINA LVII). Lupus 17(4): 314-322.

7. Hiraki LT, Benseler SM, Tyrrell PN, Harvery E, Hebert D, et al. (2009) Ethnic differences in pediatric systemic lupus erythematosus. J Rheumatol 36(11): 2539-256.

8. Tesar V, Hruskova Z (2015) Lupus Nephritis: A Different Disease in European Patients? Kidney diseases (Basel, Switzerland) 1(2): 110-118.

9. Pollak VE, Pirani CL, Schwartz FD (1961) The natural history of the renal manifestations of systemic lupus erythematousus. J Lab Clin Med 63(4): 537-550.

10. Austin HA 3rd, Klippel JH, Balow JE, le Riche NG, Steinberg AD, et al. (1986) Therapy of lupus nephritis. Controlled trail of prednisone and cytotoxic drugs. N Engl J Med 314(10): 614-619.

11. Wang LC, Yang YH, Lu MY, Chiang BL (2004) Retrospective analysis of the renal outcome of pediatric lupus nephritis. Clin Rheumatol 23(4): 318-323.

12. Gourley MF, Austin HA 3rd, Scott D, Yarboro CH, Vaughan EM, et al. (1996) Methylprednisolone and cyclophosphamide, alone or in combination, in patients with lupus nephritis. A randomized, controlled trial. Ann Intern Med 125(7): 549-557.

13. Barbano G, Gusmano R, Damasio B, Alpigiani MG, Buoncompagni A, et al. (2002) Childhood-onset lupus nephritis: a single-center experience of pulse intravenous cyclophosphamide therapy. J Nephrol 15(2): 123-129.

14. Szymanik Grzelak H, Kuzma Mroczkowska E, Maldyk J, Panczyk Tomaszewska M (2016) Lupus nephritis in children- 10 years' experience. Cent Eur J Immunol 41(3): 248-254.

15. Opastirakul S, Chartapisak W (2012) Pulse cyclophosphamide induction treatment in Thai children with diffuse proliferative lupus nephritis. Nephrology (Carlton) 17(3): 269-273. 
16. Lehman TJ, Onel K (2000) Intermittent intravenous cyclophosphamide arrests progression of the renal chronicity index in childhood systemic lupus erythematosus. J Pediatr 136(2): 243-247.

17. Houssiau FA, Vasconcelos C, D Cruz D, Sebastiani GD, Garrido Ed Ede R, et al. (2002) Immunosuppressive therapy in lupus nephritis: The EuroLupus Nephritis Trial, a randomized trial of low-dose versus high-dose intravenous cyclophosphamide. Arthritis Rheum 46(8): 2121-2131.

18. Chan TM, Tse KC, Tang CS, Mok MY, Li FK, et al. (2005) Long-term study of mycophenolate mofetil as continuous induction and maintenance treatment for diffuse proliferative lupus nephritis. J Am Soc Nephrol 16(4): 1076-1084.

19. Kise T, Yoshimura H (2012) Mycophenolate mofetil in induction and maintenance therapy for juvenile onset severe lupus nephritis. Nihon Jinzo Gakkai Shi 54(2): 86-93.

20. Sun L, Xu H, Liu HM, Zhou LJ, Cao Q et al. (2011) Long-term follow-up of 101 cases with pediatric lupus nephritis in a single center in Shanghai. Zhonghua Er Ke Za Zhi 49(11): 819-824.

21. Jin SY, Huang DL, Dang XQ, Yi ZW (2017) Lupus glomerulonephritis in 788 Chinese children: a multi-centre clinical and histopathological analysis based on 549 renal biopsies. Paediatr Int Child Health 37(4): 286-291.

22. Isenberg D, Appel GB, Contreras G, Dooley MA, Ginzler EM, et al. (2010) Influence of race/ethnicity on response to lupus nephritis treatment: the ALMS study. Rheumatology (Oxford) 49(1): 128-140.

23. Pereira T, Abitbol CL, Seeherunvong W, Katsoufis C, Chandar J, et al (2011) Three decades of progress in treating childhood-onset lupus nephritis. Clin J Am Soc Nephrol 6(9): 2192-2199.

24. Houssiau FA, D Cruz D, Sangle S, Remy P, Vasconcelos C, et al. (2010) Azathioprine versus mycophenolate mofetil for long-term immunosuppression in lupus nephritis: results from the MAINTAIN Nephritis Trail. Ann Rheum Dis 69(12): 2083-2089.

25. Tamirou F, D’Cruz D, Sangle S, Remy P, Vasoncelos C, et al. (2016) Longterm follow-up of the MAINTAIN Nephritis Trail, Comparing azathioprine and mycophenolate mofetil as maintenance therapy of lupus nephritis. Ann Rheum Dis 75(3): 526-531.

26. Dooley MA, Jayne D, Ginzler EM, Isenberg D, Olsen NJ, et al. (2011) Mycophenolate versus azathioprine as maintenance therapy for lupus nephritis. N Engl J Med 365(20): 1886-1895.

27. Contreras G, Sosnov J (2007) Role of mycophenolate mofetil in the treatment of lupus nephritis. Clin J Am Soc Nephrol 2(5): 879-882.

28. Hagelberg S, Lee Y, Bargman J, Mah G, Schneider R, et al. (2002) Longterm follow up of childhood lupus nephritis. J Rheumatol 29(12): 2635-2642.

29. Chen W, Tang X, Liu Q, Chen W, Fu P, et al. (2011) Short-term outcomes of induction therapy with tacrolimus versus cyclophosphamide for active lupus nephritis: A multicenter randomized clinical trial. Am J Kidney Dis 57(2): 235-244.

30. Li X, Ren H, Zhang Q, Zhang W, Wu X, et al. (2012) Mycophenolate mofetil or tacrolimus compared with intravenous cyclophosphamide in the induction treatment for active lupus nephritis. Nephrol Dial Transplant 27(4): 1467-1472.

31. Tanaka H, Watanabe S, Aizawa Yashiro T, Oki E, Kumagai N, et al. (2012) Long-term tacrolimus-based immunosuppressive treatment for young patients with lupus nephritis: a prospective study in daily clinical practice. Nephron Clin Pract 121(3-4): 165-167.
32. Fu LW, Yang LY, Chen WP, Lin CY (1998) Clinical efficacy of cyclosporin a neoral in the treatment of pediatric lupus nephritis with heavy proteinuria. Br J Rheumatol 37(2): 217-221.

33. Liu Z, Zhang H, Liu Z, Xing C, Fu P, et al. (2015) Multitarget therapy for induction treatment of lupus nephritis: a randomized trial. Ann Intern Med 162(1): 18-26.

34. Zhang H, Liu Z, Zhou M, Liu Z, Chen J, et al. (2107) Multitarget therapy for maintenance treatment of lupus nephritis. J Am Soc Nephrol 28(12): 3671-3678.

35. Aragon E, Resontoc LP, Chan YH, Lau YW, Tan PH, et al. (2016) Long-term outcomes with multi-targeted immunosuppressive protocol in children with severe proliferative lupus nephritis. Lupus 25(4): 399-406.

36. Tanaka H, Aizawa T, Watanabe S, Oki E, Tsuruga K, et al. (2014) Efficacy of mizoribine-tacrolimus- based induction therapy for pediatric lupus nephritis. Lupus 23(8): 813-818

37. Cortés-Hernández J, Torres Salido MT, Medrano AS, Tarrés MV, Ordi Ros J (2010) Long-term outcomes--mycophenolate mofetil treatment for lupus nephritis with addition of tacrolimus for resistant cases. Nephrology Dialysis Transplantation 25(12): 3939-3948.

38. Lanata CM, Mahmood T, Fine DM, Petri M (2010) Combination therapy of mycophenolate mofetil and tacrolimus in lupus nephritis. Lupus 19(8): 935-940

39. Nwobi O, Abitbol CL, Chandar J, Seeherunvong W, Zilleruelo G (2008) Rituximab therapy for juvenile-onset systemic lupus erythematosus. Pediatr Nephrol 23(3): 413-419.

40. Watson L, Beresford MW, Maynes C, Pilkington C, Marks SD, et al. (2015) The indications, efficacy and adverse events of rituximab in a large cohort of patients with juvenile-onset SLE. Lupus 24(1): 10-17.

41. Lehman TJ, Singh C, Ramanathan A, Alperin R, Adams A, et al. (2014) Prolonged improvement of childhood onset systemic lupus erythematosus following systematic administration of rituximab and cyclophosphamide. Pediatr Rheumatol Online J 12(1): 3.

42. Hahn BH, McMahon MA, Wilkinson A, Wallace WD, Daikh DI, et al. (2012) American college of Rheumatology guidelines for screening, treatment and management of lupus nephritis. Arthritis Care Res 64(6): 797-808.

43. Chapter 12: Lupus nephritis (2012) Kidney international supplements 2(2): 221-232.

44. Bertsias GK, Tektonidou M, Amoura Z, Aringer M, Bajema I, et al. (2012) Joint European League Against Rheumatism and European Renal Association-European Dialysis and Transplant Association (EULAR/ ERA-EDTA) recommendations for the management of adult and paediatric lupus nephritis. Annals of the rheumatic diseases 71(11): 1771-1782.

45. Pons Estel GJ, Alarcón GS, McGwin G, Danila MI, Zhang J, et al. (2009) Protective effect of hydroxychloroquine on renal damage in patients with lupus nephritis: LXV, data from a multiethnic US cohort. Arthritis and rheumatism 61(6): 830-839.

46. Rua Figueroa I, Lopez Longo J, Galindo Izquierdo M, Calvo Alen J, Del Campo V, et al. (2017) Incidence associated factors and clinical impact of severe infections in a large, multicentric cohort of patients with systemic lupus erythematosus. Semin Arthritis Rheum 47(1): 38-45.

47. Ugarte A, Danza A, Ruiz Irastorza G (2018) Glucocorticoids and antimalarials in systemic lupus erythematosus: an update and future directions. Curr Opin Rheumatol 30(5): 482-489. 
ISSN: 2574-1241

DOI: 10.26717/BJSTR.2018.11.002062

Aihua Zhang. Biomed J Sci \& Tech Res

(i) This work is licensed under Creative Commons Attribution 4.0 License

Submission Link: https://biomedres.us/submit-manuscript.php

$\begin{array}{ll}\text { BIOMEDICAL } & \text { Assets of Publishing with us } \\ \text { RESEARCHES } & \text { - Global archiving of articles } \\ & \text { - Immediate, unrestricted online access } \\ & \text { - Rigorous Peer Review Process } \\ \end{array}$

